\title{
Patron Driven Acquisitions Via Mobile Crowd Sensing
}

QianCheng $\mathrm{Yu}^{1, \mathrm{a}}$, XiaoFeng Wang ${ }^{1, \mathrm{~b}}$, Fangzheng Yang ${ }^{2, \mathrm{c}}$

\author{
${ }^{1}$ Department of Computer Science, Beifang Ethnic University, YinChuan, 750021, China \\ ${ }^{2}$ Academy of Broadcasting Science, SAPPRFT, Beijing, 100866, China \\ aemail: 570622676@qq.com, bemail: 54656337@qq.com, ${ }^{c}$ email:yangfangzheng@abs.ac.cn
}

\begin{abstract}
Keywords: SoLoMo;Patron Driven Acquisition; Mobile Crowd Sensing; Library collection development; Crowdsourcing; WeChat Application

Abstract. The concept of readers participating in the selection of library materials has been around for some time, but in recent years, with the the popularity of smart mobile phone, new opportunities for patron-based selection have emerged. In this paper, we propose a patron driven acquisitions mode via mobile crowd sensing in the support of Social, Local and Mobile technologies that named SoLoMo in short. Explored the usage of SoLoMo technologies such as participatory sensing, crowd sourcing, social recommendation, based on WeChat platform, we propose and develop a mobile application named RDASLC to collect readers' need. First, we illustrates the development process of collection development mode, then introduced the state of art of mobile library information services, especially focusing on four SoLoMo technologies: mobile crowd sensing, crowd sourcing, participatory sensing, public application development technology. The body of this paper describes in detail the analysis and design of PDAWA, emphasized to elaborate the overall framework of PDAWA, and clarifies the design thinking of simplify readers input operation and cover the needs of all readers, then analyze the three core foundation components of PDAWA, including: Bibliographic information databases, data analysis module and purchase order generation algorithm.
\end{abstract}

\section{Introduction}

According to statistics, the average circulation rate of collections in university library in our country below $40 \%$, some library even less than $20 \%$, there are about nearly 4 billion books in the "sleep" status in all the universities library of our country[1], on the other hand, the reader also often complain about can not find needed books in the library. At present, there are two prominent contradictions in university library collection construction: limited funds, but books that can been purchased are variety and price increasing, so that reader's requirements can not be satisfied and result in the loss of readers, also utilization rate of collections decline.Library collection development is the process of meeting the information needs of the people (a service population) in a timely and economical manner using information resources locally held, as well as from other organizations[2]. The widely used collection construction mode is that book list is supplied by book seller, and then decided by librarians who in charge of purchasing or who are responsible for some subject. This mode result in readers who are the eventually user of books at the passive acceptance status: readers have no right to choose which books to be purchased, can only read books which are selected by librarians. The popularity of smart mobile phone, enables us to have the condition to set up a mobile public platform through which readers can immediately submit their reading interest .This mobile application can help us to discern reader's need in the participatory sensing mode, (a.k.a. Mobile Crowd sourcing) which with lowest cost and widest scope. This kind of social recommendation application, often supported by SoLoMo methods and technologies, enable reader to submit their reading needs immediately whenever and wherever. In this way, we can easily get the requirements of readers more comprehensively, and can possible predict book demand. When used properly, participatory selecting models for patrons can support the overall collection development efforts.In this paper a new optimal design of soccer robot control system which is based on mechanical analyses and calculations on the pressure and transmutation states of chip kick mechanics, this new control system with high precision for speed control and high dynamic quality. 


\section{Library Collection Development Mode}

Collections have always been at the heart of libraries, among academic librarians, collection development has long been considered a vital duty, if not something of a nigh-sacred trust and exercise of librarians' authority and judgment[3]. Patron-Driven Acquisition has become a topic of discussion at recent meetings of library associations, several articles have been recently published and a book on the subject is appearing in early 2012[4]. Anderson et al. discuss the book on demand program of the Purdue university libraries which has been operating since 2000[5]. In this program, books requested through interlibrary loan were purchased rather than being borrowed from other libraries. It is a program that provides valuable information into the collection process, especially from graduate students. The findings of a pilot project between the University of York library and Springer are described by Thompson[6]. The best thing about selective PDA is that it will allow libraries to better gauge the needs of patrons and provide books that fit their needs. It compliments traditional collection development and the 2 together will result in a better collection overall. PDA is an abrogation of responsibility of collection development librarians. Patrons are interested only in getting the book they need now.

\section{SoLoMo Technologies}

The smart phone is turning into a "cross-technology platform", which creates new services prospects by sending local information in combination with innovative services and technologies. Smart natives are distinguished by heavy usage and a high affinity to technology and the web. They comprehensively integrate the intrinsic potential of the mobile Internet into their everyday life, the rapid development of the mobile Internet is changing people's lives.. Such "location based services" are increasingly combined with convenient perception of patron's interest, which can enable libraries provide accurate service.

SoLoMo is a comprehensive application technology, relates to mobile communication, social network, data mining, intelligent perception, wireless network, Internet of things, the semantic web, digital map, mobile location, near field communication, collective intelligence perception, cloud computing, public platform and so on . Specifically refers to the use of mobile devices, based on the geographical location and the combination of social networking (SNS) characteristics and provide network services [7]. Of course, a specific SoLoMo application does not involve all of these techniques. Collective intelligence Perception is defined as the ability of a group to solve more problems than its individual members. It is argued that the obstacles created by individual cognitive limits and the difficulty of coordination can be overcome by using a collective mental map. For problems of information transmission, storage and processing, computer technologies may come to the rescue. This has led to the creation of the field of Computer-Supported Cooperative Work (CSCW), which aims at the design of Groupware or "Group Decision Support Systems". CSCW systems can alleviate many of the problems we enumerated. By letting participants communicate anonymously via the system it can even tackle the problem of pecking order, so that all the contributions get an even opportunity to be considered. Crowd sourcing and participatory sensing are the two most common collective intelligence perception technology.

Crowd sourcing is the process of obtaining needed services, ideas, or content by soliciting contributions from a large group of people, and especially from an online community, rather than from traditional employees or suppliers[8]. While this definition from Merriam Webster is valid, a more specific definition is heavily debated. The process of crowd sourcing is often used to subdivide tedious work and has occurred successfully offline-see the examples below. It combines the efforts of numerous self-identified volunteers or part-time workers, where each contributor of their own initiative adds a small portion to the greater result. The term "crowd sourcing" is a portmanteau of "crowd" and "outsourcing"; it is distinguished from outsourcing in that the work comes from an undefined public rather than being commissioned from a specific, named group [9] . 
The proliferation of smart phones contributes to the prosperity of a new kind of sensing paradigm-mobile crowd sensing(MCS). MCS is a new sensing paradigm that empowers ordinary people to contribute data gathered or generated from their mobile devices[11]. It further aggregates heterogeneous crowd sourced data in the cloud to extract hidden intelligence (e.g., from ambient audio/visual signal to location semantics). From the AI perspective, MCS is founded on a distributed problem-solving model where crowds are engaged in complex problem solving procedures through open calls. There have been numerous MCS-powered applications, such as environment monitoring, traffic planning, social context sensing, public safety, social event replay, etc. From the perspective of data collection, these MCS applications have much in common. [10]. We Chat is a good mobile Internet technology-based application. It takes full advantage of the intelligent terminal in terms of mobility and convenience and finds the right product positioning in the numerous mobile Internet applications. It also coincides with the era of substantial improvement of the over all quality of the development of mobile Internet. The charges of data flow are continuing to decrease; the wireless network coverage and communication quality is improving significantly. These factors have provided a solid foundation for the development of WeChat. It is based on the QQ friends and cell phone contacts lists, which are convenient and effective ways of building circles of friends. Users can quickly build a social network circle in a short period of time[11]. Therefore, WeChat has gained a large number of users in a very short time.

\section{System Overview of PDAWA}

PDAWA (Patron Driven Acquisition WeChat Application) is a mobile application based on WeChat platform, we propose and develop this application to collect readers' need via mobile crowd sensing. The system architecture of PDAWA is shown in Figure 2. PDAWA is mainly composed of four functional modules, we will briefly describe its major components in the following. Purchase recommend module provide function to collect reader's needs via mobile crowd sensing, to answer the question that which book to be buy in the process of library acquisition; Vote and count module realize the function of perceive patron's interest more widely in the support of crowd sourcing, to address the issue that how many copies of one kind of book to buy; Purchase order generate module get out order list according to patron's recommendation and vote result, combined with findings carried out by data analysis module (such as history borrow pattern, reader's interest and interest community etc.), also comprehensively consider library collection constructing long term planning and the effective usage of limited fund. Data analysis module utilize the data mining technologies and social network analysis methods to process massive data set such as reader's information, history borrow/return records, history patron recommend items etc. to mining reader's interest, borrow patterns and identify community, provide a basis for the share recommended items function via social recommend ways in the purchase recommend module and the allocate vote items function in the vote and count module.

User experience is the most important feature of mobile application, PDAWA should provide simplified input function to readers because they may encounter new request any where and any time, and want to input the book items immediately. Consider the resource limited running environ-ment of mobile application, PDAWA should extremely make the users input little information word by word. Here, readers can give query keyword such as title, authors, series name, ISBN of a book to get the information of the book from backend bibliographic database. And the input methods can range from text form (Chinese word, PinYin first character, PinYin full characters), voice from, to scan book form (WeChat 5.0 provide a new function that can get book information by scan bar code or cover ). 


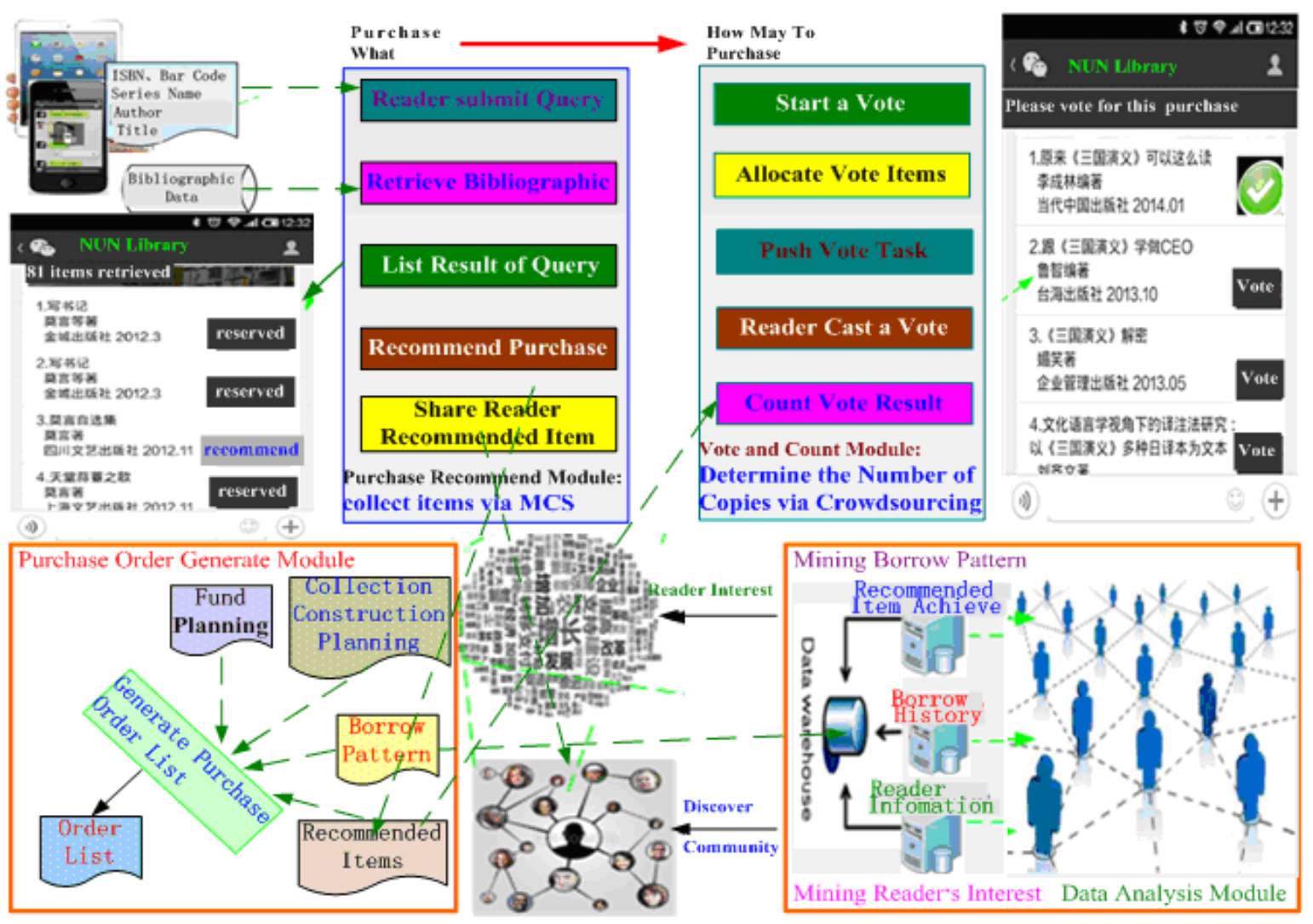

Fig.1. The system architecture of PDAWA

Patrons submit query keyword to the library application server via their smart phone and get the book information from the server. The partial process flow of this procedure is as show in figure 3:first, convert reader's input into standard format (voice form query keyword transfer to standard text form via voice recognition, bar code form transfer to standard text form by barcode identification, cover form input transfer to standard text form through image processing, and PinYin form input transfer to Chinese word form).If encounter error or ambiguous semantic during the procedure of the transfer, PDAWA will prompt user to input query key word again. PDAWA retrieve the bibliographic database with the standard query key word and display the book information to readers mobile end if the book exists in the database. Also, PDAWA will check if the book is reserved in structure to add a new attribute to indicate if the book is reserved or not, if reserved in the collection, this attribute get 1 as its value, 0 otherwise). While display the book informa-tion on the mobile phone screen, there will has an indicate sign 'reserved' if the book exist in the collection of library, otherwise, a button named 'recommend' will be appear at the tail of the record.

After all, one person's viewpoint and justification ability are limited, the key concept of crowd sensing is that based on the massive people's participatory to percept crowd behavior pattern. One person's sensing data maybe do not make sense, but when thousands and millions of person's perception data are collected together, a qualitative leap will take place. Make use of these ordinary data in proper ways, we can mining valuable information. Mobile crowd sensing expand the coverage through diversify the data collect source, social recommendation and crowd sourcing widen the range of awareness via widespread push the information. According to reader's recommendation history, readers are divided into two groups, one is called active readers group, the other is called inactive readers group. Active readers are the enthusiastic contributors of recommendation, their recommended advices often include numerous information and are of high values. To provide effective express ways to the active readers, PDAWA have a share recommendation item function in the purchase recommend module. With this function, library period push the latest recommended item to other active readers who have the similar reading 
interest with the donors, to solicit their opinions to those items. The key technology difficulties to implement this function is that we must know which items are pushed to selected active readers.

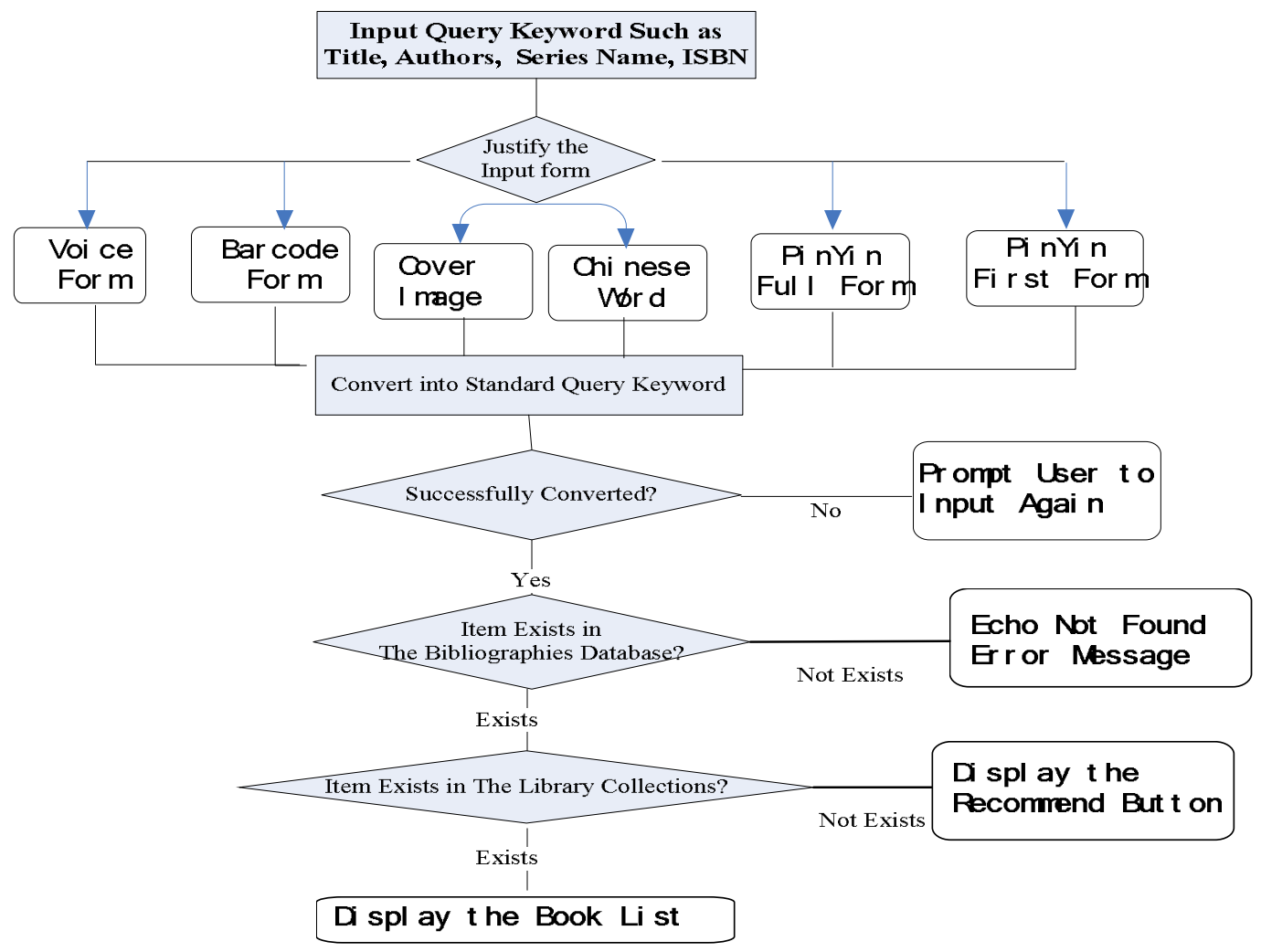

Fig.2. The partial process flow of PDAWA's book query process

In order to collect inactive readers' interest, PDAWA provide vote function in the vote and count module, librarians will regularly initiating a voting activity to vote and count the recommended items and push the items to the inactive readers. The key technology difficulties to realize this function is that we must know selected items are pushed to which inactive readers and how many selected items are be sent to on voter.

PDAWA has three key core base: bibliographic database, data analysis module, purchase order generation algorithm. The construction of library bibliographic database is the vital foundation of all library business systems. The vast majority of libraries in China establish the bibliographic database which in accordance with the CN MARC format. Objectively, the bibliographic information is inconsistent between the library, press, and book publishing authorities, mainly due to the following factors: (1) the classification of the original book is incorrect or description items are partially incomplete; (2) the selected field less or lack of certain field information; (3) some of the information in accordance to the incorrect field or some fields have wrong information; (4)wrong words or leakage recorded words caused by manual input. Bibliographic information inconsistency will seriously affect readers using PDAWA, so it should been synchronized with the bookseller's bibliographic database in time. Data analysis module is the information engine of RDAWA, implementation of many core functions (such as mining the interest of readers, reveal borrow patterns, discover book friend circles, generate common recommender list among active readers, push the most interesting potential recommend items to inactive readers, generate purchase orders based on social recommendation etc.) are basically depend on this module. The vital technical difficulties in developing data analysis module is that: how to construct affiliation network according to reader's demographic attributes ( gender, nationality, profession, grade, etc.)? How to construct social network according to the borrow history, consider with reader's knowledge structure and reading interesting? 


\section{Conclusion}

PDAWA is a crowd sourcing platform for collecting readers' interest in mobile participatory sensing pattern. Readers' interests are very extensive and dynamic change constantly with time, also, readers' demands are highly affected by their knowledge structure, professional direction, individual state or even external events can also cause readers interest and demand change (for example: Mo Yan won the Nobel prize for literature, which drives a lot of readers who don't known Mo Yan before began to read Mo Yan's books). The development of wireless communication and smart mobile devices has been the impetus for mobile crowd-sensing applications. Collection management and organization research is the area where traditional library missions and practices are reinterpreted for the mobile library environment. Progress in this area is essential if mobile library collections are to meet successfully the needs of their user communities.. The authority and quality of patron's recommendations are of central concern to the libraries. Ensuring and identifying these attributes of content calls for research that spans both technical and organizational issues. Research is also needed to clarify the roles of librarians and institutions in defining and managing collections in the mobile environment.

\section{Acknowledgment}

This work is supported by the scientific research project of the Beifang Ethnic University (Grant No.2014XYZ03,2014XYS17,2014 XBZ04) and the the National Natural Science Foundation of China (Grant No. 61462001).

\section{References}

[1] Xinhua news agency, Most university libraries in Nanjing are not open, book circulation rate of libraries in less than 10 percent nation wide, http://news.sctv.com/jyxw/201205/138510.shtml

[2] Chepesuik, R. (1997). The future is here: America's libraries go digital. American Libraries, vol.2, No.1, pp. 47-49

[3] Stowell Bracke, M.. Science and technology books on demand: A decade of patron-driven collection development, part 2. Collection Management, 35(3/4), 142-150

[4] Nixon, J. M., R. S. Freeman and S. M. Ward, Patron-Driven Acquisi-tions: Current Successes and Future Directions. Taylor and Francis, 2011

[5] Anderson, K.; R. Freeman; J-P. Herubel; L. Mykytiuk ; J. Nixon; and S.Ward, Liberal Arts Books on Demand: A Decade of Patron-Driven Collection Development, Part 1, Collection Management, Volume 35, 125-141, 2010

[6] Thompson, S., User $\square$ Driven Purchasing: a Pilot Project to Test an Alternative Pricing Model for Springer e $\square$ Book Collections, Serials: The Journal for the Serials Community, Volume 23, Number 2, 135-139, July 2010

[7] Zheng Yang, Chenshu Wu, and Yunhao Liu. Locating in Fingerprint Space:Wireless Indoor Localization with Little Human Intervention. In Proceedings of ACM MobiCom, 2012

[8] Chatzimilioudis, G., Konstantinidis, A, Laoudias C., and Zeinalipour Yazti D. Crowdsourcing With Smart Phones. IEEE Internet Comput-ing 16, 5 (2012), 36-44

[9] Ganti R K, Ye F, Lei H. Mobile Crowdsensing: Current State and Challenges. IEEE Communications Magazine 49, 11 (2011), 32-39

[10] Ganti R K,Ye F,Lei H, Mobile crowd sensing: Current state and future challenges, IEEE Communications Magazine, 2011, 49(11): 32-39

[11] Hong, L. 2012. Crm in Sport Events Communication: Taking A New Media Wechat as an Example. People's Press. pp. 1-54 